\title{
Connected Correlators in Random Geometries
}

\author{
J. Ambjørn ${ }^{a}$, P. Bialas ${ }^{\mathrm{b}, \mathrm{c}}$, J. Jurkiewicz ${ }^{\mathrm{a}, \mathrm{d}}$ \\ ${ }^{a}$ The Niels Bohr Institute, Blegdamsvej 17, DK-2100 Copenhagen Ø, Denmark \\ ${ }^{\mathrm{b}}$ Fakultät für Physik, Universität Bielefeld, 335615 Bielefeld, Germany \\ ${ }^{\mathrm{c}}$ Institute of Comp. Science, Jagellonian University, 30-072 Krakow, Poland \\ ${ }^{\mathrm{d}}$ Institute of Physics, Jagellonian University, 30-059 Krakow, Poland
}

\begin{abstract}
We analyze correlation functions in a toy model of a random geometry interacting with matter. We show that in general the connected correlator will contain a long-range scaling part which is in some sense a remnant of the disconnected part. This result supports the previously conjectured general form of correlation functions. We discuss the interplay between matter and geometry and the role of the symmetry in the matter sector.
\end{abstract}

\section{Introduction}

Simplicial quantum gravity, also called dynamical triangulations (DT), provides a simple and constructive definition of the path integral over the geometries which enables the use of MonteCarlo techniques (see [1] and references therein) and gives an opportunity to make a fully nonpertubative study of various quantities including correlation functions.

A possible reparametrization invariant definition of a two-point correlator in the canonical ensemble is [2]:

$$
G_{n}^{\mathbf{A B}}(r)=\left\langle\frac{1}{n} \sum_{i, j}^{n} \mathbf{A}_{i} \mathbf{B}_{j} \delta_{d(i, j), r}\right\rangle_{n}
$$

The average is taken over all configurations with a fixed volume $(n)$. Symbol $d(i, j)$ denotes the geodesic distance - the shortest path between the points $i$ and $j$.

The definition of the connected correlation functions is more ambiguous [2, 3]. Here we use the following formula 顿:

$$
\begin{aligned}
& G_{n}^{\mathbf{A B} \operatorname{conn}(r)=} \\
& \quad\left\langle\frac{1}{n} \sum_{i, j}^{n} \delta_{d(i, j), r}\left(\mathbf{A}_{i}-\langle\mathbf{A}\rangle_{n}\right)\left(\mathbf{B}_{j}-\langle\mathbf{B}\rangle_{n}\right)\right\rangle_{n} .
\end{aligned}
$$

The correlators (11) are very difficult to study and very few analytic results exist [A. ing body of numerical evidence suggests however the existence of a simple structure common to the correlators (11) in various ensembles of random geometries [4].

In this contribution we analyze a simple model of random geometry interacting with non-critical matter which permits the detailed analytic analysis of the correlators (11) and (2). The importance of such a study stems from the fact that finite size effects can make the non-critical correlations appear as long-range and this effect must be properly subtracted 顿.

\section{Branched Polymers}

Branched Polymers (BP) provide a very simple but non-trivial example of a random geometry ensemble [6]. Moreover they exhibit a wide range of properties in common with higher dimensional DT systems [7].

Here we consider the Ising model on a $\mathrm{BP}$ as the simplest model of matter coupled to a random geometry. The system is described by two coupled equations [8]:

$$
Z_{ \pm 1}(\mu)=e^{-\mu} \sum_{s= \pm 1} e^{s h} e^{ \pm s \beta} F\left(Z_{s}(\mu)\right)
$$

where $\beta$ is the inverse temperature and $h$ the magnetic field. For simplicity we chose $F(z)=$ $1+z+z^{2}$ which corresponds to a BP with $q_{i} \leq 3$ where $q_{i}$ denotes a number of branches in the vertex $i$. For this choice the system is always in the generic (elongated) phase with $\gamma_{s t r}=\frac{1}{2}$ and 
$d_{H}=2$.

The general form of the correlation function (11) in this model is 10 :

$$
\begin{gathered}
G_{n}^{\mathbf{A B}}(r) \approx 2 \sqrt{n} \mathcal{C}\langle\mathbf{A}\rangle\langle\mathbf{B}\rangle g\left(a_{0} \frac{r+\delta_{\mathbf{A}}^{(0)}+\delta_{\mathbf{B}}^{(0)}}{2 \sqrt{n}}\right) \\
+2 \sqrt{n} A_{1} B_{1} e^{-\mu_{1} r} g\left(a_{1} \frac{r+\delta_{\mathbf{A}}^{(1)}+\delta_{\mathbf{B}}^{(1)}}{2 \sqrt{n}}\right)
\end{gathered}
$$

where $g(x)=x e^{-x^{2}}$ and the non-negative parameters $\mathcal{C}, a_{0}, a_{1}, \mu_{1}$ depend only on $\beta$ and $h$ and are independent of the choice of operators $\mathbf{A}(\mathbf{B})$.

From (4) and (2) it follows that :

$$
\begin{aligned}
& G_{n}^{\mathbf{A B c o n n}}(r) \approx \frac{1}{2 \sqrt{n}} X_{\mathbf{A B}} g^{\prime \prime}\left(a_{0} \frac{r+\delta_{\mathbf{A B}}^{(0)}}{2 \sqrt{n}}\right) \\
&+2 \sqrt{n} Y_{\mathbf{A B}} e^{-\mu_{1} r} g\left(a_{1} \frac{r+\delta_{\mathbf{A B}}^{(1)}}{2 \sqrt{n}}\right)
\end{aligned}
$$

The parameters $X_{\mathbf{A B}}, Y_{\mathbf{A B}}$ and $\delta_{\mathbf{A B}}^{(k)}$ appearing in (5) are simple functions of the parameters in the formula (4).

When $h=0$ symmetry implies $Z_{+}(\mu)=Z_{-}(\mu)$ and the equations (3) decouple [8]. The model is solvable and we obtain :

$$
X_{\mathbf{s s}}=0 \quad \text { and } \quad Y_{\mathbf{q q}}=0
$$

We see that in this special case the matter-matter correlator (2) contains only the short-range part, while the purely geometric correlator appears as a long-range one.

In case of the non-zero magnetic field the system (3) cannot be solved analytically and the values of the coefficients $X_{\mathbf{A B}}$ and $Y_{\mathbf{A B}}$ in (5) must be evaluated numerically. We performed the calculations for $\beta=1.0$ and $h=0.1$ corresponding to $\mu_{1} \approx 0.5605$. We compared the analytical predictions with the MC simulations of systems with $n$ equal to 250,1000 and 4000 to check the validity of the large $n$ approximation.

In the figures 1 and 2 we present the results for the connected spin-spin and curvature-curvature correlations. As predicted each contains a longrange scaling and a short range non-scaling part. They retain however some "memory" of the symmetric case (6) : the matter (spin-spin) correlation function is dominated by the non-scaling

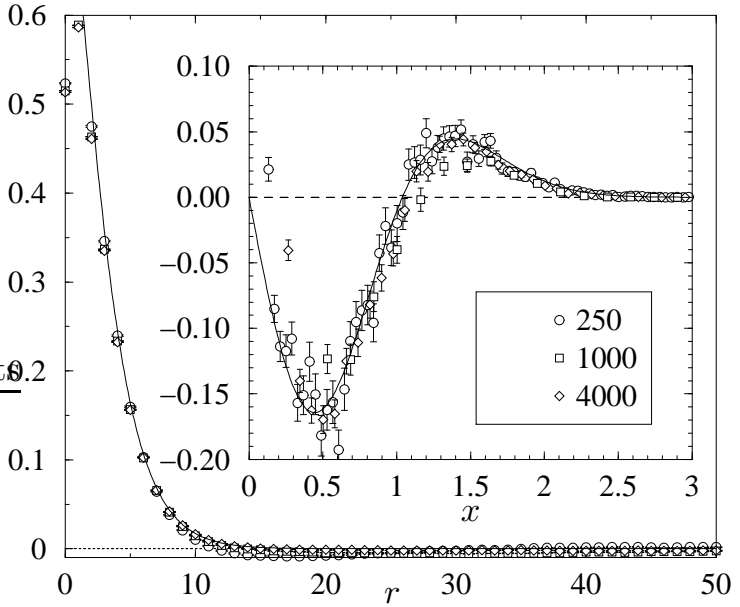

Figure 1. Spin-spin correlation function.

part and the geometric correlator is dominated by the scaling-part so that the structure (5) is not immediately visible.

To make the structure of (5) more explicit we redraw in the inlay in figure 1 the spin-spin correlators for various sizes rescaled by $2 \sqrt{n}$ as a function of the scaling variable $x=\frac{r}{2 \sqrt{n}}$.

In figure 2 we again emphasize the scaling behavior by plotting the rescaled geometric correlators on top of each other. The non scaling part can only be seen for small $r$ and large $n$ and is shown in the inlay.

In both cases the MC data are consistent with the analytic predictions shown by the continuous line.

\section{Improved correlator}

The form (4) of the correlation function suggests an improved definition of the connected correlator. For $r \gg \frac{1}{\mu_{1}}$ we have

$$
G^{\mathbf{A B}}(r) \approx\langle\mathbf{A}\rangle\langle\mathbf{B}\rangle G^{\mathbf{1 1}}\left(r+\delta_{\mathbf{A}}^{e f f}+\delta_{\mathbf{B}}^{e f f}\right)
$$

with $\delta_{\mathbf{A}}^{e f f}=\delta_{\mathbf{A}}^{(0)}-\delta_{\mathbf{1}}^{(0)}$. This relation was the source of the scaling term in the connected correlators (2). It is possible to subtract this term by 


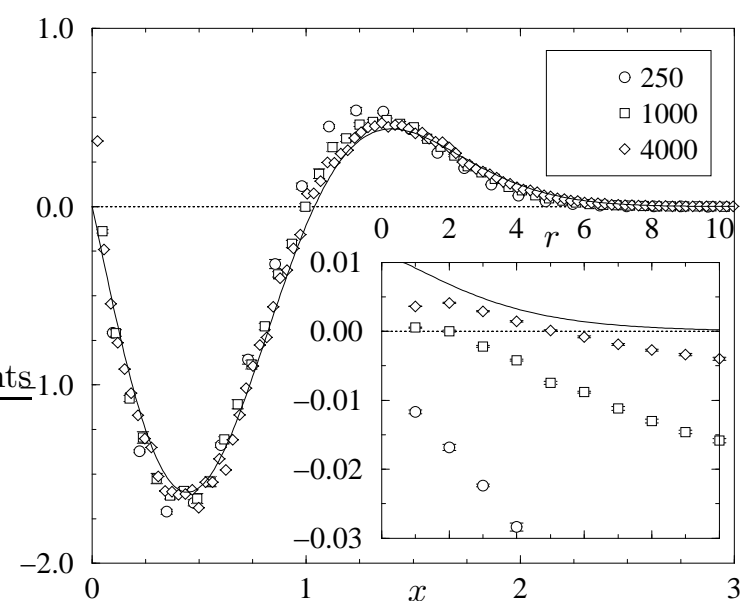

Figure 2. "Curvature-curvature" $(q-q)$ correlation function.

redefining the connected correlator to [4:

$$
\begin{aligned}
& G_{n}^{\mathbf{A B} c o n n}(r)=G_{n}^{\mathbf{A B}}(r) \\
& -\langle\mathbf{A}\rangle_{n} G^{\mathbf{1 B}}\left(r+\delta_{\mathbf{A}}^{e f f}\right)-\langle\mathbf{B}\rangle G_{n}^{\mathbf{1} \mathbf{A}}\left(r+\delta_{\mathbf{B}}^{e f f}\right) \\
& +\langle\mathbf{A}\rangle\langle\mathbf{B}\rangle G_{n}^{\mathbf{1 1}}\left(r+\delta_{\mathbf{A}}^{e f f}+\delta_{\mathbf{B}}^{e f f}\right)
\end{aligned}
$$

The values of shifts $\delta^{e f f}$ can be obtained by fitting the relation (雨) to the functions $G^{\mathbf{1 A}}$ and $G^{1 \mathrm{~B}}$ 叫.

After inserting (11) into (8) the scaling term in (2) will decrease by a factor $\frac{1}{n}$ while the nonscaling term will retain its leading order behavior.

\section{Discussion}

The obtained results can be generalized to a BP model with other types of matter and with two-point geometric interactions [10]. The main result remains unchanged : the correlator (5) contains scaling and non-scaling parts, but additional non-scaling terms appear. The result (6) can also be generalized. If the matter field takes values in a group and the partition function is symmetric under the action of this group then $X_{A B}=0$ for all matter operators (respecting the symmetry).

Numerical simulations indicate that the asymptotic behavior (7) is even more general and valid in almost every kind of random geometry en- sembles. In particular the behavior of the Ising model in a magnetic field coupled to the $2 \mathrm{D}$ simplicial quantum gravity exhibits qualitatively the same features and the improved correlator can be successfully used [4]. Similar structure is observed for the correlators of the local action density of the Abelian gauge fields in the 4D simplicial quantum gravity [11].

It seems that there exists a high degree of "universality" in the structure of correlators in systems with a random geometry. Simple models as the one described above can give us so far the unique opportunity to study this structure analytically.

The picture which emerges is the following: the purely geometric correlators (2) behave asymptotically according to (7). This leads to the appearance of the long-range correlation term in the disconnected correlator (11). The matter fields pick up these correlations through the local coupling to the geometry. Where symmetry is present this local coupling is inhibited and the long-range term is absent.

Acknowledgments P. B. was supported by the Alexander von Humboldt foundation and TMR network ERBFMRX-CT97-0122.

\section{REFERENCES}

1. G. Thorleifsson Nucl. Phys. Proc. Suppl. $\mathbf{7 3}$ (1999) 133,

2. B. V. de Bakker J. Smit Nucl. Phys. B454 (1995) 343-356,

3. P. Bialas Nucl. Phys. PS 53 (1997) 739

4. J. Ambjorn, P. Bialas, J. Jurkiewicz JHEP 02 (1999) 005,

5. H. Kawai, N. Kawamoto, T. Mogami, Y. Watabiki Phys. Lett. B306 (1993) 19-26,

6. J. Ambjorn, B. Durhuus, J. Frohlich, P. Orland Nucl. Phys. B270 (1986) 457.

7. P. Bialas, Z. Burda, B. Petersson, J. Tabaczek Nucl. Phys. B495 (1997) 463,

8. J. Ambjorn, B. Durhuus, T. Jonsson, G. Thorleifsson Nuc. Phy. B398 (1993) 568.

9. P. Bialas Phys. Lett. B373 (1996) 289-295,

10. P. Bialas in preparation.

11. J. Ambjorn, K. N. Anagnostopoulos, J. Jurkiewicz JHEP 08 (1999) 016, 